\title{
Téoros
}

Revue de recherche en tourisme

\section{La muraille ignorée ou le paradoxe de l'alliance tourisme-patrimoine en Turquie}

\section{Franck Dorso}

Volume 25, numéro 2, été 2006

Désirs d’Orient : du passé vers l'avenir

URI : https://id.erudit.org/iderudit/1071066ar

DOI : https://doi.org/10.7202/1071066ar

Aller au sommaire du numéro

Éditeur(s)

Université du Québec à Montréal

ISSN

0712-8657 (imprimé)

1923-2705 (numérique)

Découvrir la revue

Citer cet article

Dorso, F. (2006). La muraille ignorée ou le paradoxe de l'alliance tourisme-patrimoine en Turquie. Téoros, 25(2), 40-46.

https://doi.org/10.7202/1071066ar d'utilisation que vous pouvez consulter en ligne.

https://apropos.erudit.org/fr/usagers/politique-dutilisation/ 


\section{La muraille ignorée ou le paradoxe de I'alliance tourisme-patrimoine en Turquie}

\section{Franck Dorso}

En 1985, la ville d'Istanbul est inscrite sur la liste du patrimoine mondial de l'humanité par l'Organisation des Nations unies pour l'éducation, la science et la culture (UNESCO), en référence à quatre sites majeurs, dont la muraille de Théodose II. Bâtie à partir de 413, et encore bien présente sur sa partie terrestre ( $7 \mathrm{~km}$ de long, sur les 26 km du périmètre total incluant les murailles maritimes, qui ne subsistent aujourd'hui qu'à l'état de traces), l'édifice est, à partir de 1985, peu à peu dégagé des usages et des occupations qui le recouvraient, afin d'être restauré et conservé.

Ce site monumental montre un paradoxe sur le plan touristique : bien présent dans tous les guides, il n'est, sur le terrain, quasiment jamais visité. Bel exemple de construction fantasmatique, pourrait-on penser, puisque le seul point de vue direct consiste, dans le meilleur des cas, en un aperçu furtif depuis le bus ou le taxi qui file de l'aéroport vers le centre historique.

Plusieurs raisons peuvent expliquer l'échec touristique actuel. L'édifice joue un rôle historique dans la constitution physique et symbolique de la ville et il occupe aujourd'hui une position complexe dans l'aire urbaine. Le processus de patrimonialisation reste globalement problématique et met en tension des logiques, sinon opposées, du moins fondées sur des malentendus entre acteurs aux intérêts différents et changeants : municipalité, UNESCO, habitants, promoteurs, touristes, archéologues, usagers de la muraille. Ces derniers jouent un rôle de premier plan, puisqu'ils occupent concrètement les lieux. Souvent stigmatisées, ces présences révèlent des types de pratiques assez

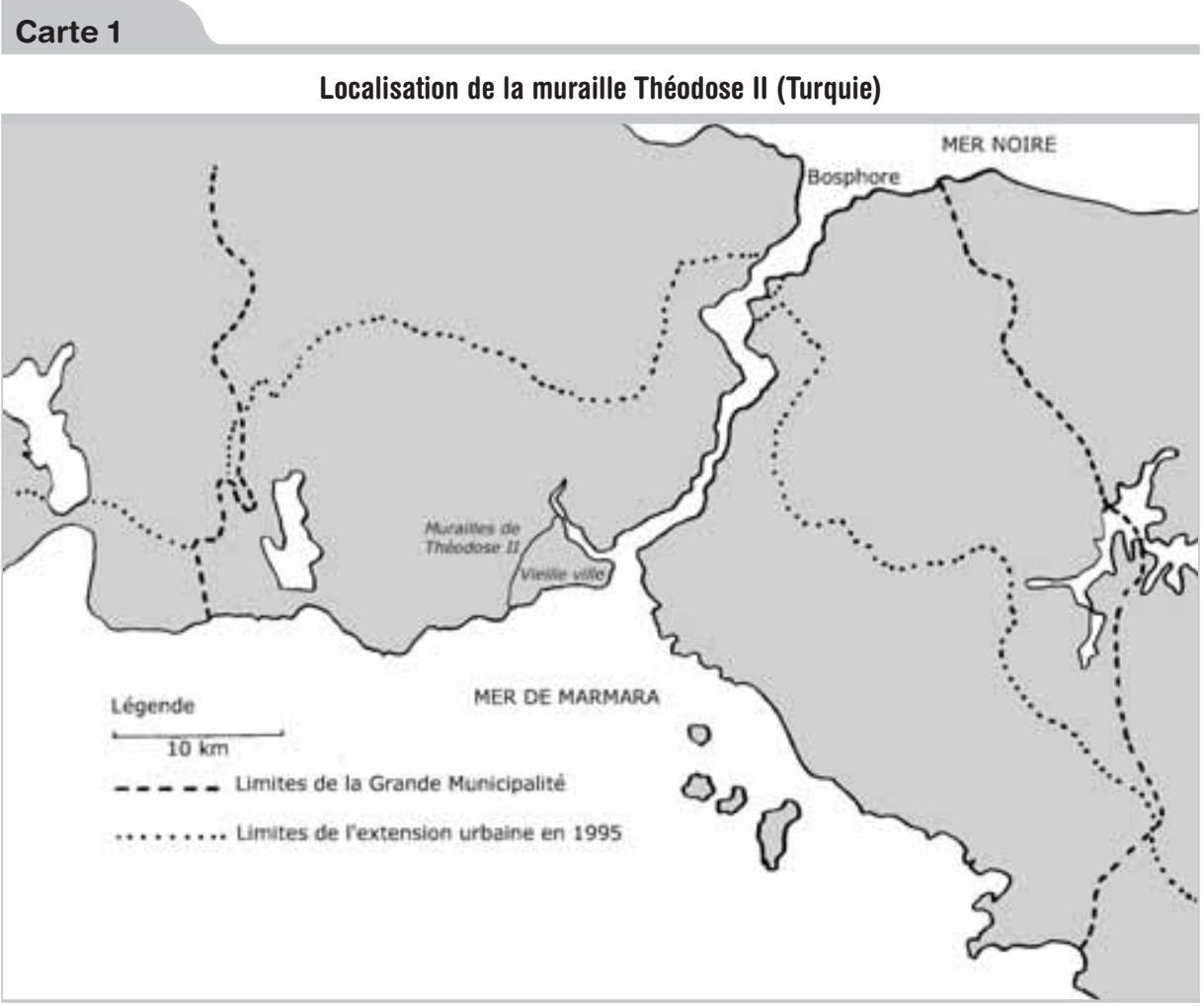

Source : Franck Dorso

différenciés, qui permettent de mieux comprendre la situation de la muraille à l'échelle de la ville et des urbanités locales.

Les récents projets touristiques pourraient bien amener une nouvelle phase de changements et montrent, en tout cas, quels arbitrages et quelles résolutions tendent à s'imposer. En devenant explicite, la question du tourisme sur la muraille permet d'envisager la rencontre - ou la friction - entre les logiques locales de l'industrie touristique et immobilière et les désirs d'Orient qui n'ont pas cessé de prendre la Turquie et la ville d'Istanbul comme des objets privilégiés.

\section{Du rempart militaire à l'héritage urbain}

La muraille que nous voyons encore aujourd'hui sur sa partie terrestre n'est pas le premier rempart défensif de l'isthme stambouliote. Le rempart de Septime Sévère, qui agrandit l'enceinte grecque primitive située à l'extrême pointe de la péninsule, protège la Byzantion du llle siècle. Constantin décide en 330 d'entourer sa future capitale d'une muraille située à 2,8 kilomètres du mur romain. En 413, l'empereur Théodose II charge le préfet Anthémius de construire un nouveau rempart, 1,5 kilomètre plus à l'ouest, qui joint 
la Corne d'Or, au nord, aux rives de la mer de Maramara, au sud, et qui sera progressivement fermé sur ses flancs maritimes (Yene et al., 2004 : 23).

Le rempart est constitué, sur sa partie terrestre, de trois murs. Le premier, en partant de l'intérieur de la ville historique, mesure 4,80 mètres d'épaisseur, pour une hauteur d'une dizaine de mètres environ. Ce mur est flanqué de 96 tours disposées tout au long de ses sept kilomètres, soit une tour tous les 50 à 80 mètres. Un espace d'une dizaine de mètres le sépare d'un second mur, d'une même hauteur mais moins épais. Devant ce dispositif est placé un avant-mur crénelé pouvant atteindre quatre mètres de haut, puis un fossé, dont le remplissage était assuré en temps de guerre par un système de digues. Ce fossé fut comblé à partir du XIXe siècle et utilisé comme lieu de production maraîchère.

La muraille participe pleinement de la construction de la ville. Monument d'exception pour l'époque, elle symbolise d'abord la puissance de l'Empire et de sa capitale. Elle pose également le cadre d'une ville et d'un empire en expansion: Théodose II place le rempart loin des dernières habitations, prévoyant et souhaitant un développement de Constantinople (Dorso, 2003 : 2). L'espace situé entre les habitations, difficilement contenues par le vieux rempart de Constantin, et la nouvelle muraille, est d'abord occupé par des champs, des vergers et des monastères, ce qui aura un impact sur les usages ultérieurs du rempart. En délimitant aussi nettement l'espace, la muraille contribue à façonner la représentation de la ville, tant pour les citadins que pour les visiteurs étrangers. Elle fait également office de frontière, sur le plan concret comme sur le plan symbolique: limite entre l'intérieur et l'extérieur, entre le plein et le vide, et même entre le vivant et le mort, puisque très tôt, et de façon durable, les pratiques funéraires placent les cimetières au pied des murailles, du côté extérieur.

À partir du XIXe siècle, l'évolution des techniques guerrières, et notamment l'apparition des canons navals, limitent l'utilité militaire de l'édifice. Celui-ci est moins entretenu, alors même que le centre de gravité de la ville se déplace sur l'autre rive de la Corne d'Or, avec la construction des palais impériaux sur les bords du Bosphore : le sultan quitte le palais de Topkap sur la pointe du Sérail pour Dolmabahçe. Dans le même temps, les activités économiques et industrielles empiètent

\section{Carte 2}

Repère de la muraille Théodose II (Turquie)

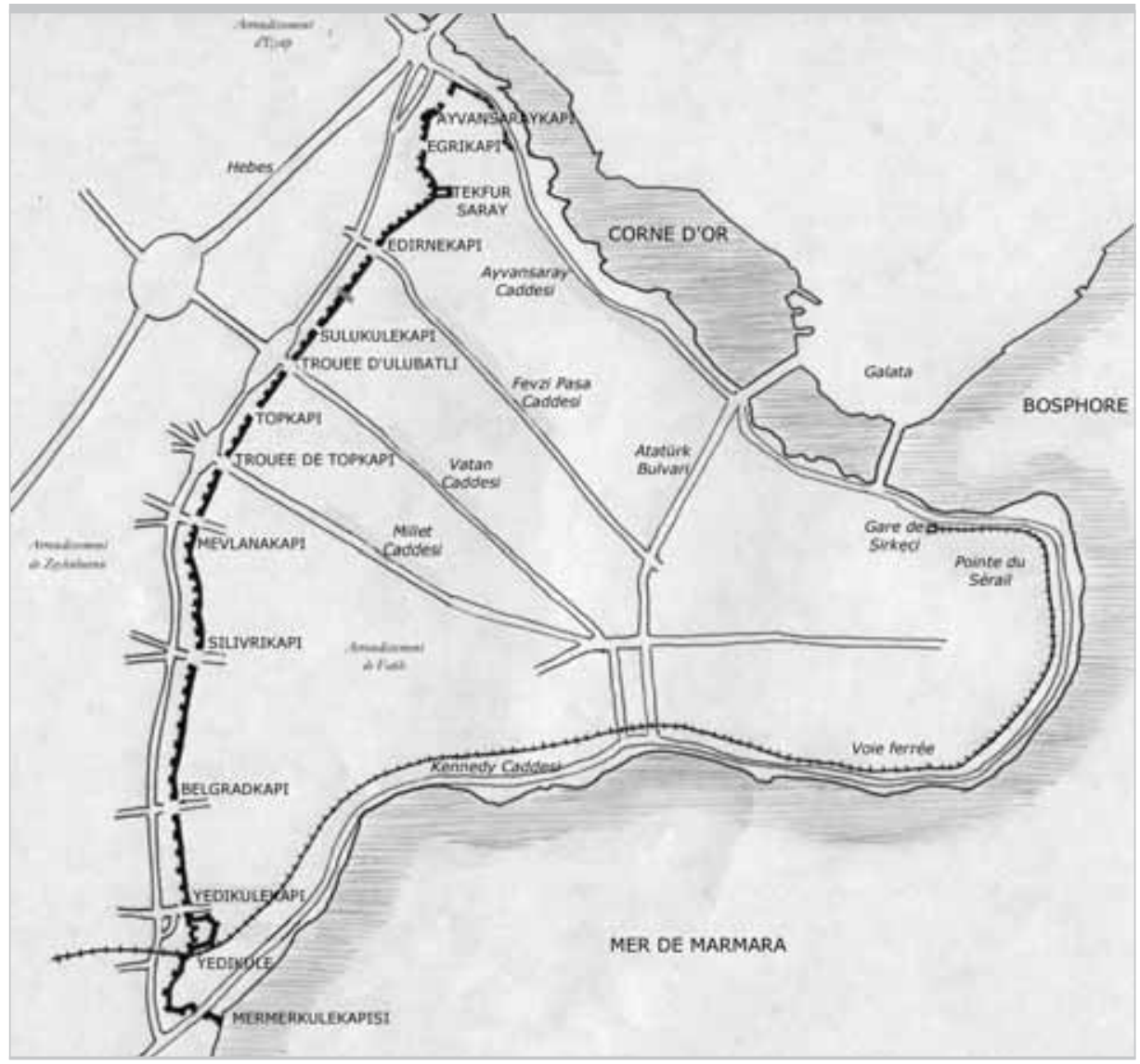

Source : Franck Dorso.

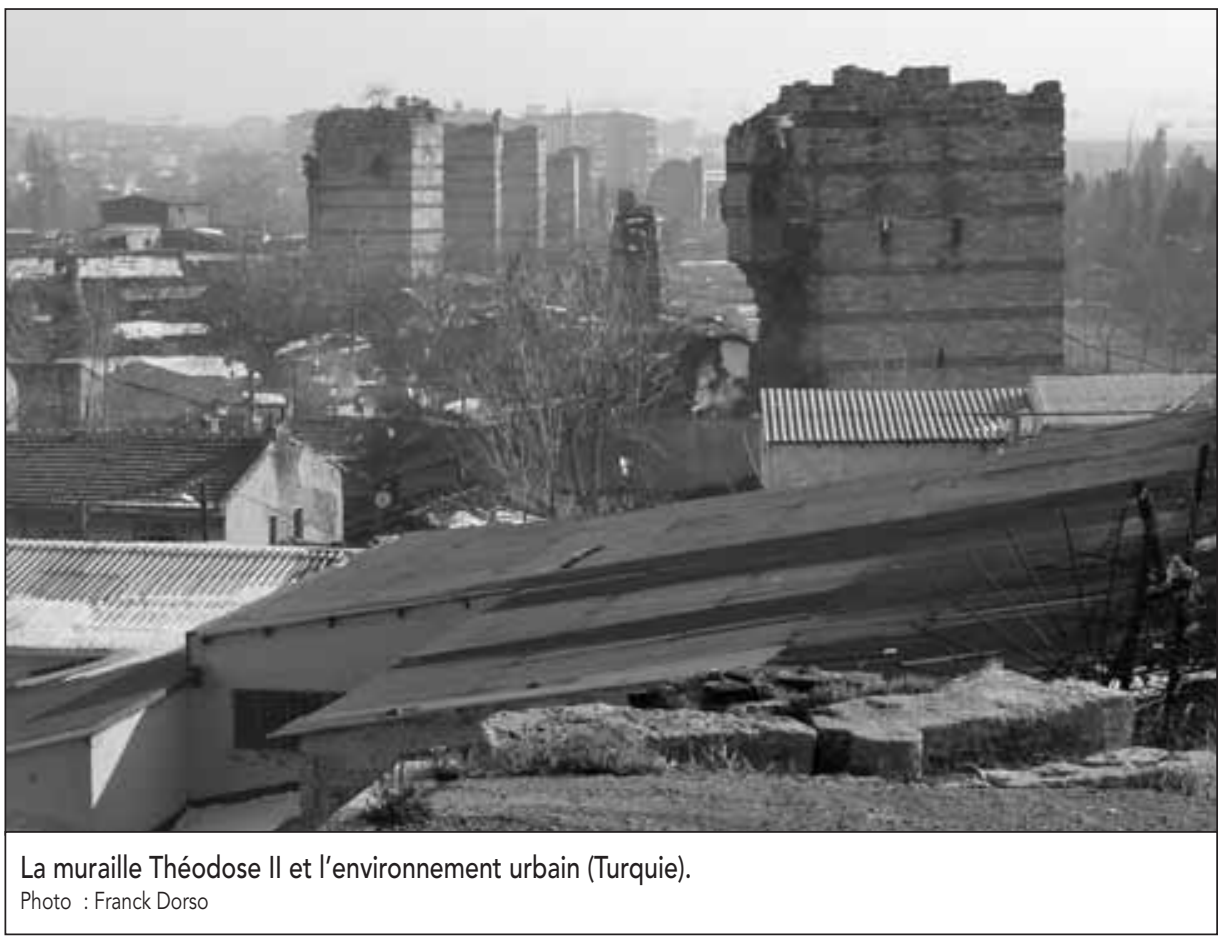


progressivement sur l'occupation résidentielle de la vieille ville. Les espaces laissés vacants sur la muraille accueillent des activités économiques qui s'installent durablement, comme les tanneries près de Yedikule, au sud. Les fossés comblés voient se développer une agriculture maraîchère organisée. Avec les explosions urbaines des années 1950 puis 1970, l'extension urbaine perce l'enveloppe du rempart. La ville s'étend sur plusieurs kilomètres et la muraille, qui définissait les limites de la ville, devient un simple édifice, noyé dans la masse.

En 1985, la ville d'Istanbul est inscrite sur la liste du patrimoine mondial de l'humanité, en référence à quatre sites majeurs, dont la muraille de Théodose II. La Turquie sort peu à peu du coup d'État militaire de 1980. Cette période, qualifiée d' " ouverture ", conforte le développement de l'industrie touristique. L'inscription patrimoniale est logiquement perçue comme une ressource importante pour ces stratégies nouvelles. Une période de changements importants commence alors sur le site de la muraille terrestre. Des opérations de dégagement et de déguerpissement ont lieu, suivies de chantiers de rénovation. Ces opérations, qui démarrent en 1985, marquent un tournant.

Très vite, des critiques s'élèvent. Les archéologues et les architectes dénoncent des travaux qui sont plus des reconstructions hâtives que des restaurations à proprement parler. Des scandales éclatent également sur l'obtention des chantiers de rénovation. À l'échelle du site dans son intégralité, aucun projet ou plan d'ensemble n'avait encadré les travaux jusqu'à cette année.

En outre, les opérations de déguerpissement n'ont pas supprimé les usages de la muraille terrestre. Lorsque les chantiers se terminent, les anciens usagers ou de nouveaux reviennent prendre possession des lieux. Les modalités des occupations durables (habitat, économie souterraine) peuvent évoluer. Ainsi, la règle de l'invisibilité se généralise. Certaines portions semblent bien dégagées, mais une visite entre les murs révèle les mêmes présences et activités qu'avant les travaux. Sur certaines portions, les usages reprennent simplement comme avant, dans un décor légèrement modifié. On observe au final, et depuis une dizaine d'années au moins, un processus de recomposition territoriale permanent (Demazière et Dorso, 2003: 18) que les autorités semblent ne pas maîtriser.
Si l'usage et l'occupation «sauvages " de la muraille de Théodose forment un tout unitaire dans les discours, et si ce fait est généralement déploré par la sphère institutionnelle, les pratiques concrètes sur le terrain montrent de la diversité et n'impliquent pas nécessairement une opposition systématique de la part des habitants des quartiers riverains.

\section{Un site touristique sans touriste?}

$\mathrm{Si}$, faute de document officiel d'ensemble sur le plan urbanistique, la vocation touristique de la muraille n'a pas été vraiment explicitée par la municipalité, cette destination transpadans les références à l'inscription patrimoniale de I'UNESCO, dans les brochures touristiques ou dans les guides turcs ou étrangers. Site majeur, très fréquemment cité, et présenté comme un tour de force presque à l'égal de la basilique SainteSophie, comme en témoigne l'usage fréquent de superlatifs, le site n'accueille pourtant qu'exceptionnellement des touristes. Aucune visite guidée n'est organisée sur la dans la plupart des cas d'un commentaire rapide lorsque le bus, venant de l'aéroport international via la Sahil Yolu (route de bord de mer) et pénétrant dans la ville historique, passe entre les vestiges de l'ancienne "porte de la tour de marbre».

La réponse la plus immédiate à ce décalage réside dans l'accessibilité du site. La muraille est d'abord éloignée du cœur vibrant de la scène touristique stambouliote, qui s'orgaraît partout de façon implicite, que ce soit muraille et les tour-opérateurs se contentent

nise autour d'un triangle formé par SainteSophie, la mosquée Bleue et le Grand Bazar. De l'arrêt de bus, tramway et taxi de Beyazıt, il faut compter environ une demi-heure (moins quand les conditions de circulation sont bonnes, un peu plus aux heures de pointe) pour rejoindre Edirnekapı, le point culminant de la muraille terrestre, qui offre une vue admirable sur la Corne d'Or, ou la station d'Ulubatlı, située au centre du rempart (considéré dans sa dimension linéaire).

Les visiteurs plus curieux, ou qui disposent de plus de temps, s'approchent pourtant parfois très près de la muraille de Théodose II : la Kariye, ancienne basilique Saint-Sauveur-in-Chora, petit édifice remarquable dont les mosaïques rivalisent avec Sainte-Sophie, n'est située qu'à quelques centaines de mètres d'Edrinekapı et du Tekfur Saray. Ceux qui prennent le temps d'arpenter la Corne d'Or vers Eyüp, en quête du site du pèlerinage voué au compagnon du prophète ou du café Pierre Loti, juché sur le promontoire surplombant l'ancien et immense cimetière musulman arboré, ne font que rarement le détour par le donjon d'Anemas, pourtant tout proche et bien visible. Les plus audacieux, qui iront, à l'opposé, manger du poisson sur les rives de la mer de Marmara et se promener sur les espaces aménagés entre la route et l'eau, pourraient être guidés par les derniers vestiges du rempart maritime pour atteindre les premières tours de la partie terrestre, mais cela s'avère encore plus rare sur cette portion.

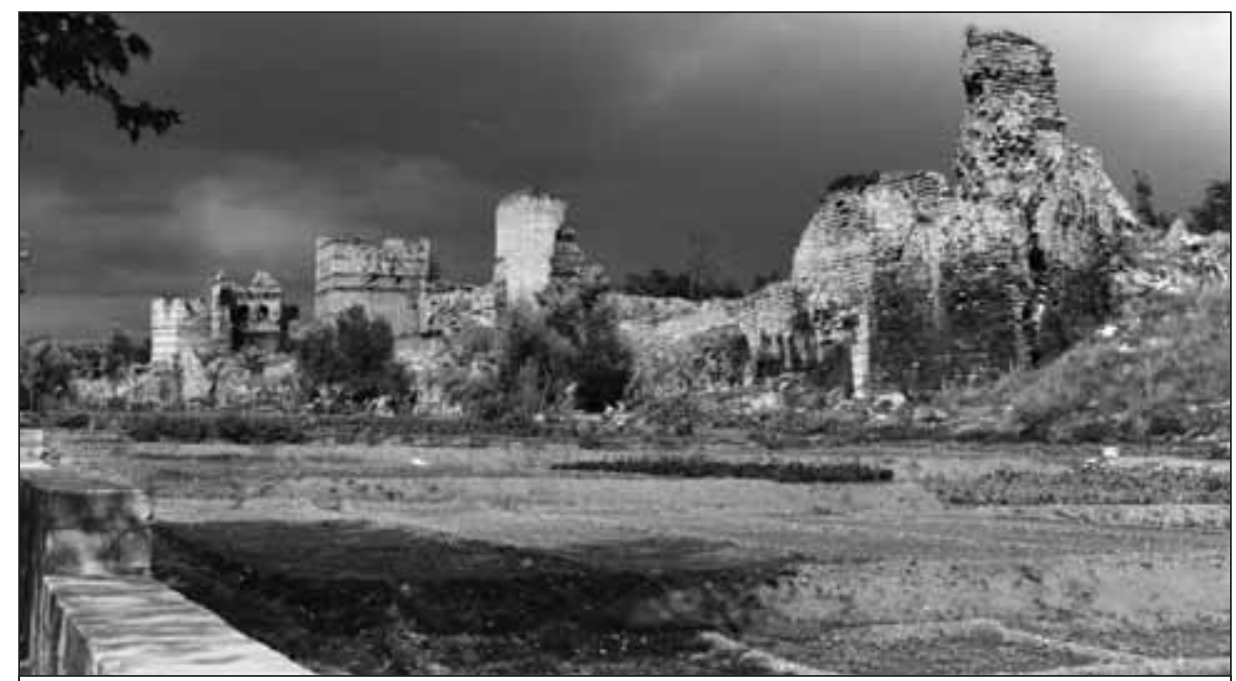

Les tours et le jardin à l'extérieur de la muraille Théodose II (Turquie).

Photo : Franck Dorso 
Seul le site de Yedikule est exploité : l'ancien dispositif militaire ottoman, siège du trésor, puis prison pour diplomates, fut restauré une première fois dans les années 1970. Au grand dam de certains archéologues turcs, il a été récemment réaménagé et une grande scène centrale accueille dorénavant des spectacles musicaux ou folkloriques. L'accès à la muraille, pourtant envisageable par l'ancienne porte d'or byzantine, monumental portique antique par lequel entraient les empereurs et qu'Anthémius intégra en 413 à la muraille, n'est pas possible. Yedikule tourne de fait le dos à la muraille de Théodose II.

Une autre raison est fréquemment évoquée pour expliquer l'absence de touristes sur le site : la muraille serait un endroit dangereux. Le risque est d'abord physique: certaines portions ébranlées par les séismes et non encore restaurées présentent des blocs de mur pouvant s'écrouler facilement. Des accidents mortels ont eu lieu, qui ont guidé la municipalité dans ses choix d'intervention. Les travaux récents et réalisés à la va-vite présentent en outre des risques d'éboulement. La brèche déjà présente en 2001 dans les travaux réalisés à la fin des années 1990 autour de la Belgradkapı s'était ainsi transformée, jusqu'en mai 2006, en un véritable écroulement, mettant en péril le tablier du mur principal ${ }^{1}$. Par ailleurs, les tuiles posées sur les rénovations de pierre et de ciment pour imiter le mode de construction byzantin ne sont pas correctement scellées et les chutes ont commencé quelques années à peine après la fermeture des chantiers.

Le risque le plus fréquemment invoqué reste toutefois l'agression - le plus souvent humaine ou par les bandes de chiens errants. Quels que soient les jugements moraux portés sur les usages de la muraille, toute présence sur un espace comporte une part d'appropriation, même labile. Les réactions hostiles peuvent alors être vues, dans certains cas, comme des défenses de territoire. Concrètement, certains usages présents sur la muraille, illégaux ou non tolérés par les habitants des quartiers voisins, viennent précisément y chercher l'invisibilité. Une arrivée impromptue sur les lieux peut facilement déclencher une réaction agressive ; c'est le cas des petits trafics ou des rencontres secrètes. Quelques incidents peuvent également survenir avec les personnes ivres. L'accueil, d'abord cha-

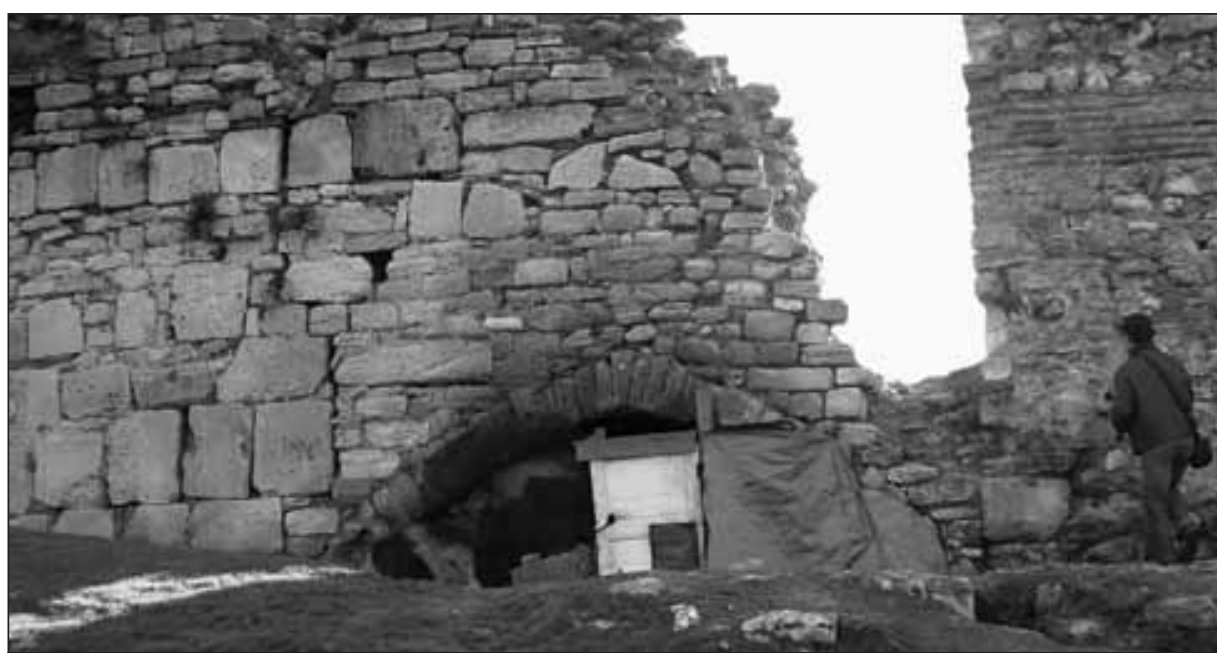

Un abri de fortune à l'intérieur de la muraille Théodose II (Turquie).

Photo : Franck Dorso

leureux, peut être suivi d'une certaine agressivité vis-à-vis du convive ou du visiteur lorsque celui-ci tente de se dégager du groupe et de partir. Les tinerci (" sniffeurs » de colle) peuvent avoir des réactions imprévisibles ou jouer aux détrousseurs (ce qui n'est évidemment pas spécifique à la muraille). Au printemps 2001, un groupe de jeunes adolescents avait pris possession du donjon d'Anemas, seul site approché par quelques rares touristes, et réclamaient avec une certaine rudesse un droit d'entrée pour pouvoir pénétrer dans les impressionnants souterrains de l'ancien palais des Blachernes. Une certaine politesse ou prudence reste de mise lorsqu'on approche d'un squat aménagé, d'une baraque de jardinier ou d'un campement rom, approche qui ne déclenche le plus souvent que des grognements de chiens. Car en effet, dans la plupart des cas, les registres d'attitude ou d'interaction prennent la forme de l'inattention polie ou des salutations courtoises. C'est même fréquemment le cas dans les endroits parfois glauques réservés aux pratiques cachées, solitaires, ou aux haltes de démunis (sommeil dans des trous de mur ou de végétation, petit feu et repas, libation).

L'insécurité généralisée, présentée à la fois dans les guides touristiques étrangers et dans les discours institutionnels ou des habitants riverains, semble donc ne pas résister à l'épreuve des faits. Certes, des violences peuvent survenir sur la muraille, mais ne caractérisent pas un endroit porteur d'une violence endémique, spécifique. Quelques mesures de prudence et une connaissance minimale du terrain permettent de parcourir l'ensemble de l'édifice presque d'un bout à l'autre, sans encombre. Le danger sur la muraille renvoie finalement à des réalités autant qu'à des fantasmes. Les violences fantasmées de la muraille véhiculées par les discours des habitants proviennent sans doute de conflits d'usages au quotidien, qui peuvent être exacerbés à certains moments (reconquêtes après les chantiers, arrivée de nouveaux venus...). L'attitude erratique de l'institution municipale sur cette question, entre laisser-faire et expulsions radicales, semble plus énigmatique et amène à élargir l'analyse au processus encore difficile de patrimonialisation de l'édifice.

\section{De l'héritage au patrimoine}

Comment interpréter en effet le manque manifeste d'intérêt porté, au moins jusqu'en 2005, par la municipalité à la muraille de Théodose II ? Aucun projet d'ensemble n'a été décidé pour l'édifice et les travaux sont engagés sans logique d'ensemble, sans attention particulière pour la qualité architecturale (Demazière et Dorso, 2003 : 18). Les critiques les plus dures évoquent la "disneylandisation". Des conflits ont opposé partis politiques et administrations municipales et nationales, à travers leurs services déconcentrés sur le terrain. II faut attendre les récriminations de I'UNESCO ${ }^{2}$ en 2003, qui menace de retirer la ville de la liste du patrimoine mondial pour la placer sur celle des chefs-d'œuvre en péril, ainsi que les mobilisations d'universitaires et d'ONG (organisations non gouvernementales), turcs et étrangers, pour voir les discours évoluer sensible- 
ment. Mais le plan au $1 / 5000^{e}$ de sauvegarde de la péninsule historique élaboré en réponse par la municipalité soulève rapidement de nouvelles critiques ${ }^{3}$.

La notion même de patrimoine pose problème. Le mot n'existe pas dans la langue turque, qui utilise le terme miras, qui signifie héritage. La plupart des urbanistes et des architectes turcs rencontrés pour discuter ce sujet pointent la relative nouveauté de ces préoccupations patrimoniales en Turquie et les décalages entre les représentations d'institutions comme I'UNESCO et celles des élus et des représentants des administrations turques chargées de la protection des sites. L'institution étatique en charge de ces questions, le Haut Conseil pour la protection des biens naturels et culturels, qui émane du ministère de la Culture et du Tourisme, inclut, comme son nom l'indique, les sites aussi bien naturels qu'architecturaux. Sur le plan municipal, un conflit larvé a opposé au début des années 2000 la direction de la construction et celle de la protection de l'environnement historique, dans un contexte d'urbanisation accélérée où les préoccupations de conservation des sites anciens n'ont pas toujours été une priorité.

L'essor du tourisme, dans les années 1960, mais surtout après 1980, repose en partie sur la mise en valeur de sites architecturaux imbriqués dans les tissus urbains denses des centres historiques de plusieurs grandes villes. Ces premières mesures de protection montrent un clivage, qui semble jouer encore un rôle aujourd'hui, entre les édifices ottomans et les édifices byzantins. Certains discours nationalistes tenus dans les années 1970 opèrent un tri sévère entre ce qui relève de «notre histoire » et le reste, qui n'est pas digne d'intérêt. On peut ainsi évoquer le projet de raser les murailles - motivé également par l'appétit spéculatif immobilier pour la vaste surface foncière ainsi dégagée - ou celui, récurrent, de refaire de Sainte-Sophie une mosquée ${ }^{4}$.

Le traitement de la muraille est donc lui aussi travaillé par ces tensions. On peut s'en rendre compte dans l'attitude paradoxale du maître d'ouvrage, la municipalité du Grand Istanbul (IBB). On observe en effet, d'un côté, un désintérêt manifeste dont nous avons indiqué les grandes lignes (en termes de projets, de travaux, de communication sur l'image), et, de l'autre, un mo- ment de surinvestissement symbolique, un seul jour dans l'année, chaque 29 mai, pour commémorer, lors d'une mise en scène grandiose et médiatisée rassemblant public, acteurs de la reconstitution, élus et défilés militaires et scolaires, la conquête de la ville par le sultan Mehmet le Conquérant en 1453. La seule appropriation du site réside ainsi dans une représentation guerrière et ponctuelle, célébrée depuis 1953, et la création de l'association de la conquête, dans une période de montée en puissance des mouvements nationalistes. La muraille, ignorée toute l'année et violentée lors d'un bref moment d'exutoire, représenterait-elle une part refoulée de la ville (Dorso, 2003 : 11)?

\section{La muraille habitée : repoussoir ou ressource?}

Sur le terrain, la " déprise » institutionnelle, qui prévalait jusqu'en 2005, a permis aux usages informels ou clandestins de la muraille de se maintenir, en s'adaptant aux opérations ponctuelles de dégagement et aux travaux.

D'une façon générale, il ne faut pas imaginer un conflit structuré entre usagers et institutionnels. Les usagers sont multiples et ne forment pas un groupe mobilisé unifié. Par ailleurs, ce sont surtout les institutions internationales ou les architectes et les universitaires qui font de la muraille le cœur du problème. Ce type d'usage informel et spontané existe dans bien d'autres endroits de l'aire métropolitaine. II est d'ailleurs étonnant de voir que les représentations spatiales de la muraille sont plutôt communes aux usagers locaux et aux représentants de la municipalité et s'opposent à l'expertise internationale ou universitaire. L'analyse des cartes mentales montre que là où les experts extérieurs considèrent un site linéaire dans son ensemble, les usagers et les pouvoirs locaux prennent davantage en compte des points - situés en des endroits stratégiques pour la mairie (du point de vue de la sécurité ou de l'image), ou situés pour les usagers dans des réseaux de lieux liés au quotidien (déplacements, promenades, courses...), et dans lesquels la muraille n'est qu'un élément parmi d'autres. En relativisant la vivacité des conflits, en confrontant les usages quotidiens aux représentations, on voit la situation se complexifier : le processus de recomposition territoriale permanent existe bien, mais s'accompagne de certaines formes de stabilité.
Comment interpréter cela du point de vue de la problématique touristique? Une certaine forme de stabilité semblait prévaloir avant 1985 : des artisans et des quartiers informels installés durablement, dans lesquels les modes de régulation du vivre-ensemble au quotidien et des espaces communs ne diffèrent pas fondamentalement d'un petit quartier classique. Après 1985, ces usages traditionnels font place à des changements, lors desquels les frictions liées à la « recolonisation » des espaces perdus, à la conquête de nouveaux espaces ou à l'arrivée de nouveaux usagers ou résidants, sont plus fortes. Mais le retour des usages et des occupations amène à nouveau, peu à peu, des modalités de régulation des interactions au quotidien. Ce n'est sans doute pas un hasard si les réflexions sur la qualité d'espace public de la muraille commencent à apparaître. Une part du sentiment de sécurité que l'on éprouve en parcourant la majorité des quartiers stambouliotes, quel que soit leur standing, provient notamment de la densité d'occupation. En termes d'interactions, des endroits vides représentent une baisse du contrôle social. Vider la muraille de tous ses usagers supprimerait les modalités actuelles d'interaction qui fonctionnent quand même au quotidien, vaille que vaille. Ne faut-il pas alors laisser aux usagers et aux résidants actuels ce rôle invisible, mais si fondamental, pour garantir l'accessibilité d'une espace dont la plus grande partie peut être qualifiée de public? En ce sens, les usages illégaux de la muraille ne font plus figure de repoussoir, mais, associés à la mise en place de parcours reconnus et acceptés par tous (sur la base des sentiers existants, par exemple), d'une ressource réelle pour assurer l'accès à l'édifice.

Cette hypothèse appelle deux réserves. Premièrement, la visite de la muraille peut devenir le support d'une forme de voyeurisme, un tourisme du sordide, de l'exploit exploratoire dans des "bas-fonds " supposés, et présentés comme tels a posteriori par les discours, les photographies. En second lieu, le simple aménagement de trajets n'apporte en soi aucune solution à certaines situations de grande pauvreté qui peuvent être rencontrées sur la muraille. Bien sûr, l'aménagement du site n'a pas la fonction d'une politique macroéconomique de réduction des pauvretés urbaines, mais ne peut-on imaginer des bénéfices pour les usagers et les résidants de la muraille? On retombe alors, pour les cas les plus difficiles, sur une situation classique de double bind : proposer un petit bénéfice 
localisé peut avoir pour effet d'enkyster les occupations les plus précaires et de les éloigner d'une mobilité résidentielle ou professionnelle plus valorisée. On pourrait finalement imaginer une résolution de ces tensions contradictoires dans une ouverture des procédures décisionnelles, à travers la concertation et la participation des usagers. Un vœu pieux assez classique, mais qui présente l'avantage de resituer la problématique dans le champ politique.

\section{Les nouveaux projets touristiques, entre muséification et opérations immobilières}

Ces solutions paraissent peu réalistes aujourd'hui, lorsqu'on les confronte avec les nouveaux projets portés par la municipalité. Un projet déjà évoqué au début des années 2000 a le vent en poupe: parfaire la rénovation de quelques points remarquables de la muraille, transformer les tours proches en boutiques et développer l'hôtellerie de proximité. La muraille prend pleinement son rôle d'outil, de ressource de l'industrie touristique, dans un contexte porteur pour les grands projets immobiliers. Istanbul sera en effet capitale culturelle européenne en 2010 et cette opportunité mobilise beaucoup d'énergie, d'institutions et d'acteurs. De grands projets voient le jour, comme la transformation de la gare asiatique d'Aydarpaşa en centre commercial tourné vers la mer et entouré de sept tours imposantes. Quoiqu'il en soit, le projet touristique pour la muraille prévoit des expulsions définitives et, dans certains cas, l'installation de sociétés de sécurité privées autour des points aménagés.

Ces projets peuvent aussi porter une dimension symbolique, par exemple celui de reconstituer un " quartier turc » au pied des murailles, dans le secteur d'Ayvansaray, non loin de la Corne d'Or. De vieilles maisons ottomanes en bois, rescapées des nombreux incendies qu'a connus la ville, mais en très mauvais état, abritaient des familles pauvres et des activités liées à la tonte et au filage de la laine. Les expulsions sont presque terminées. Les maisons seront rasées et des reconstructions prendront leur place, pour créer une vitrine mimusée mi-commerce de ce qu'est un quartier turc traditionnel. Ce type de construction nécessitera des arbitrages esthétiques et urbanistiques importants, avec des enjeux forts en termes de construction identitaire et d'écriture de l'histoire urbaine. rend entre la municipalité et I'UNESCO. Si, après les récriminations de 2003, on a pu penser que la mairie cherchait à donner le change et à reconquérir les faveurs de l'institution internationale, notamment avec le plan de sauvegarde au $1 / 5000^{\circ}$ de la péninsule historique, les bruits de couloir et les discours de moins en moins officieux des représentants de la municipalité montrent une inversion de la tendance. Pour ces derniers, il vaut mieux rompre avec les injonctions patrimoniales (tant pis si l'UNESCO place Istanbul sur la liste du patrimoine en péril !) et exercer un pouvoir souverain sur l'urbanisation de ces zones sensibles. Cette situation découle en partie de l'hégémonie du parti au pouvoir, l'AKP (parti de la justice et du développement), qui dirige maintenant à la fois le gouvernement et la municipalité du Grand Istanbul. II est possible, par ailleurs, d'évoquer la tendance à l'entrepreneurialisme urbain, à condition d'apporter quelques précisions relatives à la situation turque. L'intégration du public et du privé est ancienne, l'État kémaliste de la première moitié du $X X^{e}$ siècle ayant mis sous sa coupe le secteur entrepreneurial. Les liens sont restés forts et flous, dans des configurations qui ont évolué : le pôle entrepreneurial de la sphère du pouvoir joue aujourd'hui un rôle déterminant dans la
Ces arbitrages risquent de creuser le diffé-

définition des objectifs et des modalités des opérations urbaines, toujours guidées par la puissance publique pour des sites comme la muraille.

D’une façon générale, l'investissement municipal dans la muraille était, jusqu'à aujourd'hui, plus porté vers une communication sélective (célébration de la conquête) et la mise en scène (rénovation grandiose de quelques portes, entrées de ville) que vers l'accessibilité touristique. Cela semble aujourd'hui en train de changer. La muraille reste un site byzantin dont la prise en compte soulève des motivations contradictoires, mais elle représente surtout une opportunité économique importante en termes immobiliers. Elle pourrait ainsi devenir le support d'un tourisme culturel ou de luxe. Cette perspective oblige à assurer un niveau de prestation élevé et à ne pas dénaturer complètement le site. Les travaux de rénovation ont ainsi été arrêtés au début de cette année ${ }^{5}$ et des grilles ont été installées sur l'ultime portion achevée, autour d'Edrinekapı, pour en interdire tout accès.

Paradoxalement, en risquant de briser les liens avec I'UNESCO, la municipalité semble développer un projet de mise en valeur assez proche des canons internationaux du «patrimoine » : accessibilité sécurisée par

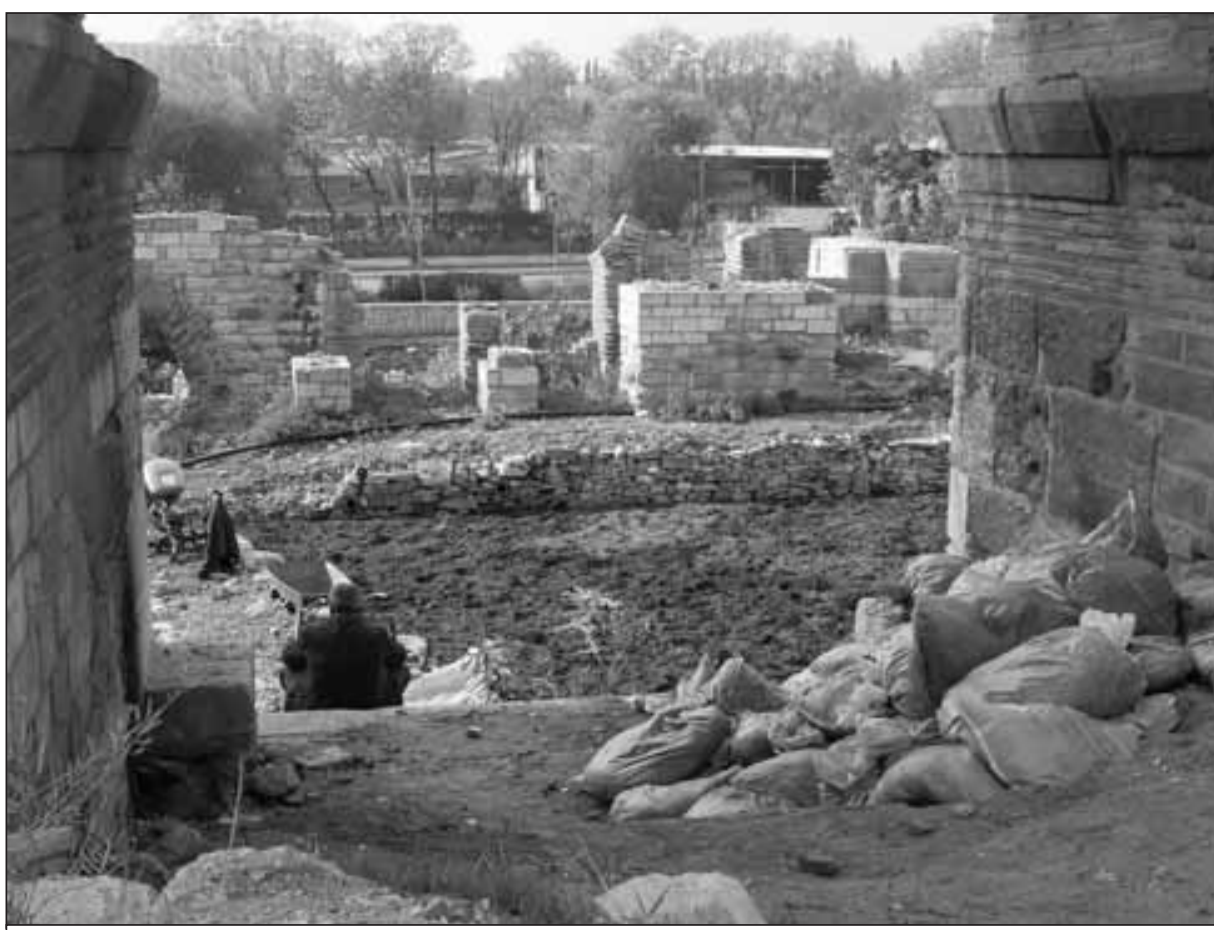

Le manque de muséification de la muraille Théodose II (Turquie).

Photo : Franck Dorso 
des gardiens, muséification du site, articulation avec l'activité commerciale et hôtelière. Ces choix s'intègrent dans d'autres choix à l'échelle métropolitaine, qui ont fait de la "régénération urbaine » (kentsel dönüşüm) un axe prioritaire. Sur l'ensemble de la péninsule historique et du secteur de Galata, dans l'actuel arrondissement de Beyoğlu, cette régénération se traduit par une gentrification sans surprise. Signe que la métropole est définitivement européenne, ou bien traduction généralisée des effets uniformisateurs de la globalisation économique dans le champ urbain?

\section{Désorientaliser la muraille, la ville ?}

Autant d'éléments qui risquent d'émousser notre « désir d'Orient »... Mais le désir est aussi un processus d'objectivation, de création de l'objet du désir. On pourrait bien, après tout, analyser notre désir d'Orient sans y mettre les pieds, en considérant simplement l'orientalisme comme un miroir de nos représentations, un support de projections, une altérité révélatrice. Dans le même temps, si le désir crée l'objet, alors il est urgent de se rendre sur place, pour confronter nos discours réifiants à la vie sociale, aux interactions locales ; en d'autres termes, envisager l'impact de nos discours, puis de nos visites, sur les situations locales. S'il n'y a toujours presque aucune présence touristique physique sur la muraille, les effets de l'intérêt et de la visite potentielle du site sont réels sur le terrain, que ce soit pour les usages sociaux de la muraille ou les configurations urbaines des zones proches. C'est semble-t-il dans cette perspective qu'il faut considérer l'écart paradoxal entre la présence immuable de la muraille dans les guides touristiques, les travaux d'archéologues ou encore les documents de I'UNESCO, et l'absence de visiteurs dans les faits.

Il faudrait par ailleurs, en confrontant nos désirs à la réalité, se garder de toute critique hâtive à propos des rénovations ou des usages du site: là où l'européocentrisme voit un objet-muraille unitaire et cohérent, la réalité locale peut en faire un simple terrain de survie économique, segmenté et resitué dans les territoires du quotidien, ou encore un obstacle à des solutions de désengorgement urbain. Les modes de conscientisation du site peuvent différer sensiblement, et notre définition linéaire et unitaire est une façon d' " essentialiser », de naturaliser un objet urbain pris dans des représentations, des images et des usages contradictoires. À l'inverse, une relativi- sation radicale présenterait les mêmes dangers : le groupe de jeunes "gardant » l'entrée du donjon d'Anemas avait bien compris la construction patrimoniale du site et le bénéfice qu'ils pouvaient en tirer. Finalement, dans la prise en compte, le « saisissement » de l'édifice, quelles légitimités entrent en confrontation ? Lesquelles privilégier? Peut-on envisager de les faire dialoguer? Quels seraient alors les processus de légitimation autorisant telle ou telle personne à venir s'asseoir à la table des discussions?

Le désir d'Orient lui-même mériterait d'être interrogé, et pas seulement comme altérité révélatrice. Istanbul est aussi, en effet, désirée de l'intérieur, par les Turcs et les Stambouliotes eux-mêmes. Les films populaires des années 1970 montrent la figure de l'immigré rural anatolien découvrant la grande ville, magnifiée et crainte à la fois, et qui finit par devenir objet de désir, médium d'intégration. La plaquette de présentation de la célébration de la conquête de 2002 assimile l'entrée dans la ville de Mehmet le Conquérant à une union amoureuse avec la cité féminisée (Pérouse, 2003). L'écrivain Adman Özyalçiner intitule un recueil de nouvelles Sur en 1967 (sur signifie muraille et désigne ici la muraille terrestre), tandis que plusieurs photographes s'y intéressent, tels Ara Güler ou Arif Aşcı. En 2003, plusieurs universitaires ou acteurs associatifs se mobilisent, à la suite des menaces de déclassement de I'UNESCO, et créent le mouvement de "Mobilisation pour la sauvegarde des remparts d'Istanbul. » Les activités nocturnes et festives sur la muraille gardent encore chez certains intellectuels l'image d'un folklore stambouliote très vivace dans les années 1950, lors de la première explosion urbaine. La ville et ses édifices sont ainsi l'objet de désirs et de fantasmes multiples, tant intérieurs qu'extérieurs. Faut-il alors, pour prendre la mesure de la situation, commencer par " désorientaliser » la ville et la muraille?

Ce travail de prise de conscience consisterait alors, pour le touriste visiteur, à se frotter au réel, à abandonner son désir d'Orient, les images et les usages qu'il souhaite trouver sur le site, pour aborder une succession de quartiers, en partie formels, en partie informels, au sein desquels émerge un élément urbain - fondamentalement urbain - réapproprié, vivant, en transformation. Loin d'une muséification tournée vers un passé souvent sélectif et toujours construit, le visiteur verrait sous ses yeux l'articulation du « passé-présent-avenir » d'une urbanité stambouliote en mouvement. Cette approche romprait, d'un côté, avec la visite touristique marchande classique et, de l'autre, avec le voyeurisme du baroudeur pseudojournaliste. Elle devrait aussi pouvoir limiter la pression sur le site occasionnée par ces présences "extraordinaires » du visiteur extérieur. Mais serait-ce encore du tourisme?

Franck Dorso est chercheur associé au laboratoire Réso de l'Unité mixte de recherche - Centre national de la recherche scientifique - Espaces et Sociétés et chargé d'enseignement à l'Université de Haute Bretagne.

\section{Notes}

1 Le visiteur qui accède au rempart par un escalier éloigné ne peut soupçonner le vide de plus de un mètre de large qui creuse le sol sous ses pieds que lorsqu'il arrive au-dessus, en constatant l'éboulement du mur en contrebas, au niveau des jardins maraîchers.

2 Qui n'a, dans le jeu des acteurs locaux et dans les actions concrètes de sauvegarde, qu'un rôle consultatif.

3 Simple plan de zonage pour les uns, ouverture à l'entrepreneurialisme immobilier pour d'autres (Ozel, 2004 : 8).

4 Journal quotidien Radikal, 25 juin 2005, p. 4, cité dans la revue de presse d'Electroui no 21, 30 juin 2005, p. 29, revue électronique de l'Observatoire urbain d'Istanbul, IFEA (Institut français d'études anatoliennes), Istanbul.

5 Sur la muraille elle-même. Mais un chantier continue au Tekfur Saray, dont une partie des murs est intégrée au rempart, pour en faire un site de spectacle, dans la veine de Yedikule.

\section{Bibliographie}

Demazière, Christophe, et Franck Dorso (2003), "Deux expériences urbaines aux marges de l'Europe ", Les Annales de la recherche urbaine, n 93, Plan Urbanisme Construction Architecture, Ministère de l'équipement, Paris, p. 15-21.

Dorso, Franck (2003), « Un espace indécis au cœur d'Istanbul », Les dossiers de l'IFEA (Institut français d'études anatoliennes), série "Patrimoines au présent », n 1, juin, 39 p.

Ozel, Derya (2004), Politiques urbaines et patrimonialisation : Quelle représentation de l'héritage architectural ? L'exemple de la péninsule historique Eminönü-Fatih, Istanbul, Rapport d'étude, Observatoire Urbain d'Istanbul, Institut français d'études anatoliennes.

Pérouse, Jean-François (2003), « La muraille terrestre d'Istanbul ou l'impossible mémoire urbaine ", Rives nord-méditerranéennes n 16, p. 27-44.

Pérouse, Jean-François (2004), La Turquie en marche, Paris, Éditions de La Martinière.

Yene, Zekiye, Nilgün Erkan Biçer, et Eser Yücetürk (2004), "Transformation of the Meaning of the City Walls of Istanbul ", Europa Nostra, vol. 58, p. 21-30. 